\title{
Diffuse Sclerosing Variant of Papillary Thyroid Carcinoma with Concomitant Thyrotoxicosis: A Case Report and Literature Review
}

\author{
Jessie Y. W. Chan'1, David K. K. Tsui ${ }^{1}$, Polly Tsui ${ }^{2}$, Tang Chung Ngai ${ }^{1}$ \\ ${ }^{1}$ Department of Surgery, Pamela Youde Nethersole Eastern Hospital, Hong Kong, China \\ ${ }^{2}$ Department of Pathology, Pamela Youde Nethersole Eastern Hospital, Hong Kong, China \\ Email: jccyw@hotmail.com
}

Received 7 February 2014; revised 5 March 2014; accepted 12 March 2014

Copyright (C) 2014 by authors and Scientific Research Publishing Inc.

This work is licensed under the Creative Commons Attribution International License (CC BY).

http://creativecommons.org/licenses/by/4.0/

(c) (i) Open Access

\begin{abstract}
Diffuse sclerosing variant of papillary thyroid carcinoma (DSVPC) is a rare variant of conventional papillary thyroid carcinoma which was first reported in 1985. It appears to be more aggressive than conventional type of papillary thyroid carcinoma. We present a case in a 14-year-old girl who initially presented with thyrotoxicosis and was later diagnosed with DSVPC on subsequent ultrasound and fine need leaspiration when cervicallymph node was evident on clinical examination. Concomitant thyrotoxicosis in thyroid carcinoma is uncommon. The prevalence, clinical presentation, characteristic radiological and histological features, management and prognosis of DSVPC are discussed with literature review. We also hope to illustrate through this case that ultrasound as a non-invasive imaging modality is an invaluable tool for workup in functional thyroid problems and concomitant malignancy may not be that uncommon, so that we could provide prompt treatment to our patients.
\end{abstract}

\section{Keywords}

Papillary Thyroid Carcinoma; Diffuse Sclerosing Variant; Thyrotoxicosis; Microcalcifications; Ultrasound

\section{Introduction}

Diffuse sclerosing variant of papillary thyroid carcinoma is a rare variant of conventional papillary thyroid car-

How to cite this paper: Chan, J.Y.W., et al. (2014) Diffuse Sclerosing Variant of Papillary Thyroid Carcinoma with Concomitant Thyrotoxicosis: A Case Report and Literature Review. Surgical Science, 5, 117-121.

http://dx.doi.org/10.4236/ss.2014.53021 
cinoma. We present a case in a young girl with concomitant thyrotoxicosis which is uncommon. The prevalence, clinical presentation, characteristic radiological and histological features, management and prognosis of the disease are discussed.

\section{Case Presentation}

First, confirm that you have the A 14-year-old girl presented with progressively increasing neck swelling with hand tremor. She enjoyed good past health and did not receive any neck irradiation before. Her paternal cousin was diagnosed with some thyroid problem more than 20 years ago. Physical examination revealed a firm diffuse goiter with mild degree of thyrotoxic eye signs. Initial blood test showed free thyroxine level of $43.5 \mathrm{pmol} / \mathrm{L}$ and thyroid stimulating hormone (TSH) level $<0.01 \mathrm{mIU} / \mathrm{L}$. Antithyroglobul in level titre was 1:400 whereas antimicrosomal antibody titre was $<1: 100$. She was treated as thyrotoxicosis with antithyroid drugs. She was rendered euthyroid with normal free thyroxine level $13.4 \mathrm{pmol} / \mathrm{L}$ after seven months of treatment. However, she was found to have enlarged right neck lymph nodes upon follow-up visit. Ultrasound scan of her neck showed enlarged right lobe of thyroid with multiple whitish calcifications scattered in the whole lobe. There was an irregular ill-defined hypoechoic shadow in the upper pole with mild increased vascularity (Figure 1(a)). The left lobe was also slightly enlarged with similar scattered calcifications. There were multiple enlarged right lower cervical lymph nodes, size up to $2.5 \mathrm{~cm}$, with similar calcification pattern as in the thyroid gland (Figure 1(b)). Ultrasound-guided fine needle aspiration of the right thyroid gland and right cervical lymph node showed malignant thyroid cells suggestive of papillary carcinoma and right cervical lymph node metastatic thyroid carcinoma respectively.

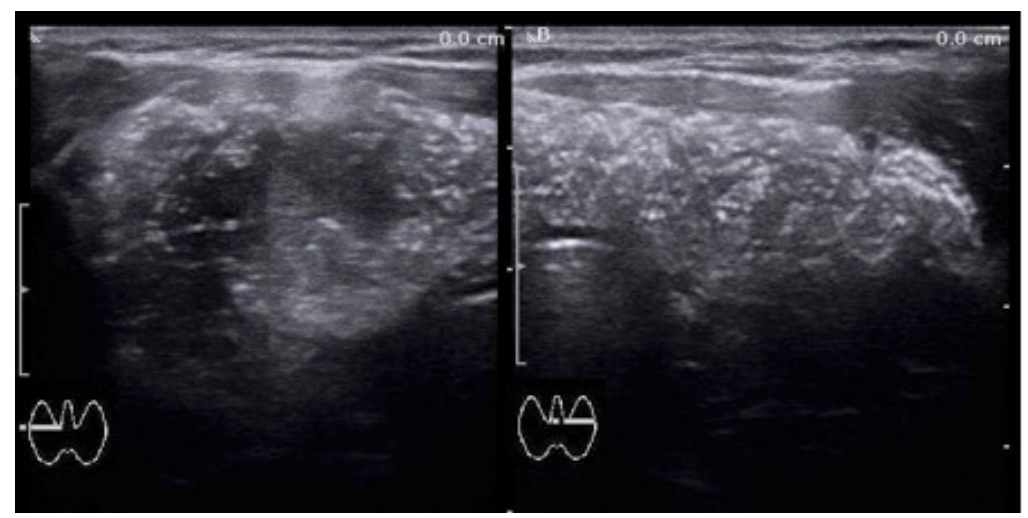

(a)

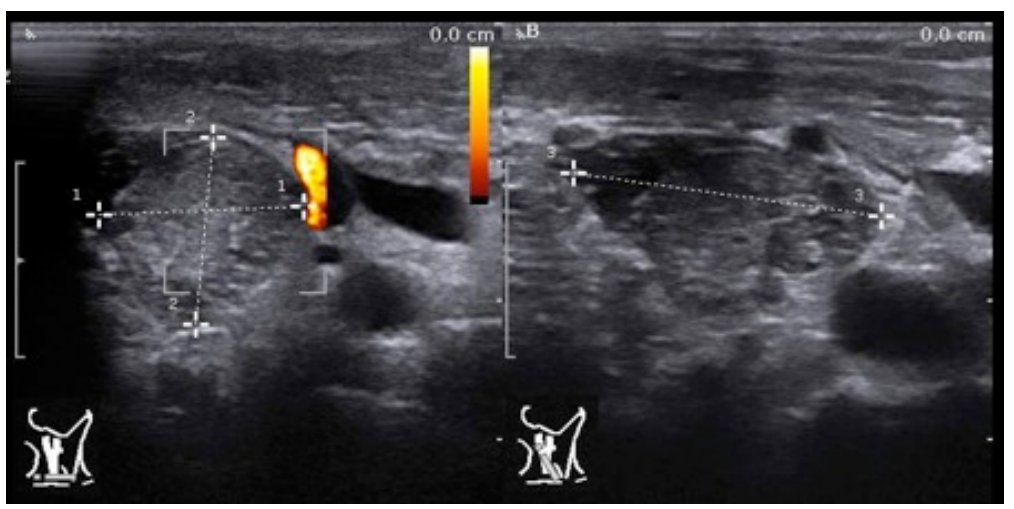

(b)

Figure 1. (a) Pre-operative ultrasound showed diffuse scattered microcalcifications over both lobes of thyroid with ill-defined hypoechoic shadow in right lobe. (b) Right lateral neck lymph node showed enlargement with tiny microcalcification similar to the thyroid parenchyma. Needle aspiration confirmed metastatic thyroid carcinoma. 
Open total thyroidectomy together with right functional neck dissection was performed. A hard tumor was noted in the right lobe intra-operatively with multiple enlarged cervical lymph nodes over level II, III, IV and VI, with largest lymph node at level III up to $2.5 \mathrm{~cm}$ in size. Pathological examination revealed a 58 gram thyroid gland with asymmetrical enlargement of the right lobe. Macroscopic examination showed an ill-defined and unencapsulated solid whitish tumor nearly replacing the whole right lobe. The left lobe was grossly not involved. Microscopic examination found the tumor diffusely infiltrated the right lobe and isthmus and less extensively the left lobe. The main tumor in the right lobe was composed of papillary proliferation with multiple psammoma bodies and small foci of squamoid solid and follicular pattern (Figure 2(a)). Invasion of fibrous capsules with tumor foci on the capsular surface were noted in both lobes. Lymphovascular infiltration was seen in both thyroid tissue and capsule. The remaining thyroid tissue was compatible with adenomatous hyperplasia. Overall features were compatible with papillary carcinoma of diffuse sclerosing variant. Twenty-nine out of 46 resected lymph nodes showed metastatic papillary carcinoma, morphologically similar to the main tumor (Figure 2(b)). Intravascular extranodal spread was noted. TMN staging was pT3N1b.

The patient received radioactive iodine ablation with dose of 3.77GBq around one month after the operation. Post-ablation whole body scintigraphy found suspected remnant thyroid tissue and/or residual tumor in the thyroid bed with multiple bilateral cervical lymph node metastases, also diffuse mild radioiodine activity over bilateral lung fields suspicious of microscopic lung metastases. TSH-stimulated thyroglobulin level was elevated to $395 \mathrm{ng} / \mathrm{mL}$. Subsequent contrast CT neck and thorax showed multiple lymphadenopathies at right level II-III with largest size up to $1.1 \times 1.2 \times 2.0 \mathrm{~cm}$, left supraclavicular level and right mediastinum concerning of nodal recurrence. No frank mass was noted at the thyroid bed and there was no CT evidence of lung metastasis. Oncologist suggested excision in view of relatively sizable lymph nodes which may not be adequately covered by radioactive iodine. Second operation for right functional neck dissection and selective left neck dissection was performed around two months after the first operation. Enlarged right upper jugular lymph nodes up to $1.5 \mathrm{~cm}$ in size at level IIb and III, with enlarged left supraclavicular lymph nodes up to $1 \mathrm{~cm}$ in size were identified intraoperatively. Microscopic examination revealed metastatic papillary carcinoma in 8 out of 23 lymph nodes. The morphology was similar to that of the main tumor previously excised. Ultrasound scan was repeated around three months after the second operation, which showed no enlarged mass in the thyroid bed and no enlarged lymph nodes along right side of neck. Shotty lymph nodes up to $3.7 \mathrm{~mm}$ in size were noted over the left mid jugular chain, which were too small for needle aspiration. Follow-up post-ablation scan six months after radioactive iodine showed no scintigraphic evidence of recurrent disease or metastasis. Currently it is 3 years after the

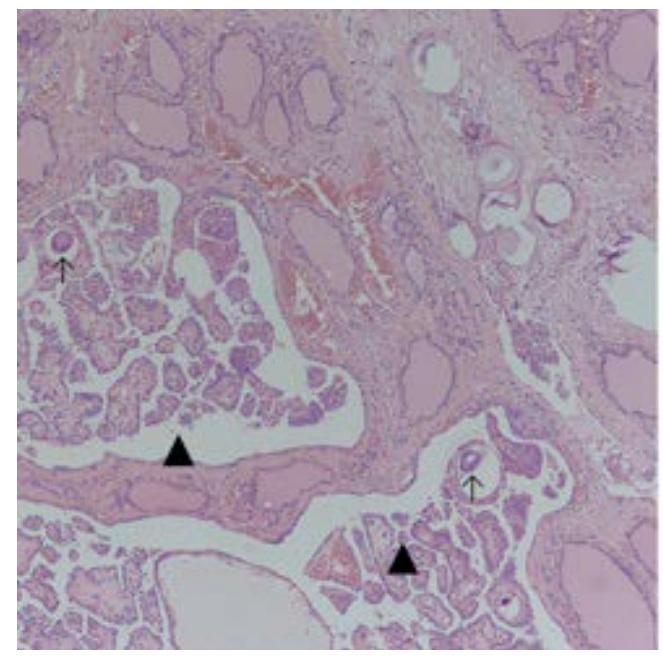

(a)

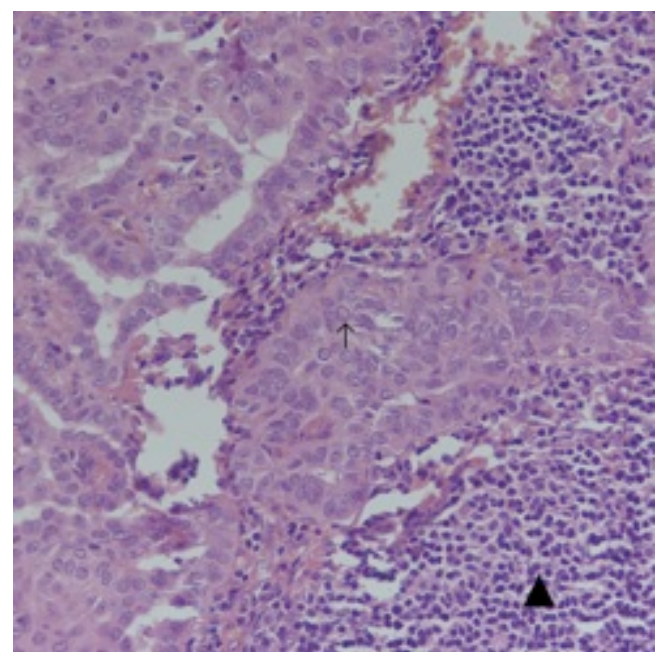

(b)

Figure 2. (a) Diffuse sclerosing variant of papillary carcinoma. The thyroid glands showed diffuse infiltrate of multiple small tumor nests, some within empty spaces (arrow heads) and presence of psammoma bodies (arrows) and lymphoid aggregates. (b) Metastasis in lymph nodes. The tumor nests are embedded in the lymphoid tissue (arrow head) and the tumor remains similar to the thyroid tumor forming nests or papillary with psammoma bodies and nuclear features of papillary carcinoma including intranuclear pseudo inclusion (arrow). 
index operation. Serial thyroglobulin level monitoring and screening ultrasound did not reveal evidence of recurrent disease. The patient is on long-termthyroxine and calcium replacement with calcium carbonate and calcitriol.

\section{Discussion}

The diffuse sclerosing type of papillary thyroid carcinoma (DSVPC) is a rare variant of conventional papillary thyroid carcinoma (PTC) first reported in 1985 [1]. The prevalence of this variant among all the patients with PTC ranged from $0.74 \%$ to $5.3 \%$ as reported in larger series [2]. It has a higher prevalence in young women, a higher incidence of lymph node and pulmonary metastasis, and a larger tumor size compared with conventional PTC [2]-[5]. It is also associated with frequent local recurrence [3] [4]. Retrospective studies of 18 to 40-year period for patients diagnosed with DSVPC presented with painless thyroid nodule or a diffuse goiter, with or without associated symptoms such as hoarsness, pressure effect, clinically detectable cervicallymph nodes, or distant metastasis [2]. Antithyroglobulin antibody level was more likely to be positive in DSVPC compared to classical PTC patients [2] [4]. Most patients are euthyroid on presentation [6] [7].

Concomitant hyperthyroidism in thyroid carcinoma is not commonly seen, although reports ranging from $0.2 \%$ to $21 \%$ of differentiated thyroid carcinoma patients with hyperthyroidism [7]. Most cases were incidental carcinoma or microcarcinoma $(<1 \mathrm{~cm})$. The true incidence of concomitant thyrotoxicosis in DSVPC is uncertain because of small number of reported cases.

Ultrasound scanning of DSVPC shows diffuse scattered microcalcifications and underlying heterogeneous hypoechogenicity, with or without association of multiple, suspicious hypoechoic and mixed echoic masses with internal microcalcifications [5].

DSVPC should be considered as a differential diagnosis in young patients with these imaging findings. Our patient showed these typical features of DSVPC (Figures 1(a) and (b)). Further investigations including guided needle biopsy should be warranted. The characteristic histological features of diffuse involvement in one or both thyroid lobes with dense fibrosis, extensive squamous metaplasia with morphologically benign nuclei, patchylymphoid infiltration with germinal centres and large number of psammoma bodies give another important clue towards the diagnosis of this disease [2]-[5].

The prognosis of DSVPC is controversial. In some earlier reported series, patients with DSVPC were reported to have an unfavorable prognosis due to extensive involvement of the thyroid, a higher incidence of cervical lymph node metastasis, and on the basis of the frequent occurrence of adverse pathologic features [4] [5]. In Japanese series, the unavailability of routine use of radioactive iodine may increase the local recurrence in their series [4]. However, some recent studies found that the prognosis of the patients with DSVPC seemed to be comparable with that of classic PTC, which may be accountable by the aggressive treatment protocol at these institutions [2]. Thus, patients with DSVPC should be treated aggressively. Initial radical surgery including active search for lymph node involvement with radical lymph node dissection followed by radioiodine treatment are justified [3] [4]. This group of patients should be monitored closely during follow up for any disease recurrence or metastasis.

\section{Conclusion}

DSVPC is a rare variant of conventional PTC. It has characteristic features on ultrasound and histological examination. The patients usually present with painless thyroid nodule or diffuse goiter and most are euthyroid. Careful initial physical examination is mandatory, followed by early ultrasound scan and/or fine needle aspiration cytology if any suspicion arises. Aggressive treatment and follow-up protocol are warranted for DSVPC for its hostile behavior. Furthermore, ultrasound as a non-invasive investigation tool should be considered in every case of functional thyroid disease as concomitant malignancy may not be that uncommon to avoid delay in treatment.

\section{References}

[1] Vickery, A.L., Carangiu, M.L., Johannessen, J.V. and Sobrinho-Simoes, M. (1985) Papillary Carcinoma. Seminars in Diagnostic Pathology, 2, 90-100.

[2] Chow, S.M., Chan, J.K.C., Law, S.C.K., et al. (2003) Diffuse Sclerosing Variant of Papillary Thyroidcarcinoma- 
Clinical Features and Outcome. EJSO, 29, 446-449. http://dx.doi.org/10.1016/S0748-7983(03)00005-2

[3] Lam, A.K.Y. and Lo, C.Y. (2006) Diffuse Sclerosing Variant of Papillary Carcinoma of the Thyroid: A 35-Year Comparative Study at a Single Institution. Annals of Surgical Oncology, 13, 176-181. http://dx.doi.org/10.1245/ASO.2006.03.062

[4] Fukushima, M., Ito, Y., Hirokawa, M., Akasu, H., Shimizu, K. and Miyauchi, A. (2009) Clinicopathologic Characteristics and Prognosis of Diffuse Sclerosing Variant of Papillary Thyroid Carcinoma in Japan: An 18-Year Experience at a Single Institution. World Journal of Surgery, 33, 958-962. http://dx.doi.org/10.1007/s00268-009-9940-6

[5] Kwak, J.Y., Kim, E.K., Hong, S.W., et al. (2007) Diffuse Sclerosing Variant of Papillary Carcinoma of the Thyroid: Ultrasound Features with Histopathological Correlation. Clinical Radiology, 62, 382-386. http://dx.doi.org/10.1016/j.crad.2006.11.015

[6] McElvanna, K., McCusker, G. and Stirling, I. (2007) Diffuse Sclerosing Variant of Papillary Thyroid Carcinoma-A Rare Cause of Goiter in a Young Patient. Ulster Medical Journal, 76, 113-114.

[7] Omur, O., Yazc, B., Akgun, A., et al. (2008) Concomitant Hyperthyroidism in Patients with Thyroid Carcinoma and the Effects of Iodine Supplementation in an Iodine-Deficient Area. Clinical Nuclear Medicine, 33, 769-772. http://dx.doi.org/10.1097/RLU.0b013e318187eeb2 